\title{
Possible Influence of Arctic Oscillation on Winter Visibility over Eastern China
}

\author{
Yu Gu1, Xiaomeng Shi2 ${ }^{*}$, Jilin Sun ${ }^{1}$ \\ ${ }^{1}$ Physical Oceanography Laboratory, Ocean-Atmosphere Interaction and Climate Laboratory, Ocean University of China, \\ Qingdao, China \\ ${ }^{2}$ Qingdao Engineering Technology Research Center for Meteorological Disaster Prevention, Qingdao Meteorological Bureau, \\ Qingdao, China \\ Email: *shi198710@126.com
}

How to cite this paper: Gu, Y., Shi, X.M. and Sun, J.L. (2017) Possible Influence of Arctic Oscillation on Winter Visibility over Eastern China. Journal of Geoscience and Environment Protection, 5, 56-62.

https://doi.org/10.4236/gep.2017.58006

Received: April 19, 2017

Accepted: July 29, 2017

Published: August 1, 2017

Copyright $\odot 2017$ by authors and Scientific Research Publishing Inc. This work is licensed under the Creative Commons Attribution International License (CC BY 4.0).

http://creativecommons.org/licenses/by/4.0/

\begin{abstract}
NCEP/NCAR reanalysis data, the Arctic Oscillation (AO) index and the atmospheric visibility (Vis) data at 134 sites in eastern China (ECN) are used to investigate the possible influence of $\mathrm{AO}$ on the wintertime weather and $\mathrm{Vis}$ over ECN. A higher relative humidity ( $\mathrm{RH}$, compared with the normal year) is identified over ECN in the winters with a positive phase of $\mathrm{AO}$, and the wind anomaly is generally inshore in the coast areas of China. All these processes are consistent with Vis degradation over ECN. On the contrary, in the winters with a negative phase of $\mathrm{AO}$, a lower $\mathrm{RH}$ can be identified over ECN, and the wind anomaly is generally offshore, which is favorable for Vis improvement.
\end{abstract}

\section{Keywords}

The Arctic Oscillation, Eastern China, Winter, Atmospheric Visibility

\section{Introduction}

In the winter of the northern hemisphere, the Arctic is usually controlled by the low-pressure system, and the mid-latitudes are usually controlled by the highpressure system. The difference between the Arctic and the mid-latitudes is called the Arctic Oscillation [1] [2] [3] [4]. AO can be divided into positive phase and negative phase [5]. When $\mathrm{AO}$ is in the positive phase, the air pressure difference between the Arctic and mid-latitudes is high, which is not conducive to the Arctic cold air southward transmission. On the contrary, the regional pressure difference of the Arctic and mid-latitudes is low with AO in the negative phase, so that the Arctic cold air is easier to expand to the mid-latitudes [6] 
[7]. Arctic Oscillation intensity has obvious seasonal changes; the winter is the season with the most significant AO mode and the strongest variability [8] [9].

As an important indicator of the quality of the atmospheric environment, Vis has a great impact on traffic and people's daily lives [10]. There are many factors influencing the winter Vis over ECN. In addition to the anthropogenic factors such as the atmosphere pollutants emissions of factories, it has close links with meteorological factors such as RH and wind field [11]. On the one hand, there is a negative correlation between Vis and RH. The lower RH leads to the improvement of Vis [12]. This is because the more water vapor in the atmosphere is conducive to the hygroscopic growth of the aerosol particles, and the water vapor itself absorbs light and scattered light [13]. On the other hand, the Vis will be improved when the wind field is conducive to the proliferation, dilution and sedimentation of atmospheric particles.

When the AO is in the negative phase, the cold air in winter Arctic is easy to spread southward, leading to the occurrence of cold tide [6] [7]. The weather is usually windy and dry with cold tide [13]. The dry air is not conducive to the formation and hygroscopic growth of the aerosol particles and strong winds is conducive to the diffusion and sedimentation of atmospheric pollutants, so that the atmospheric Vis improves. On the contrary, when the AO is in the positive phase, the cold air in winter Arctic is not easy to spread southward, and the cold tide occurs less [6] [7]. So the humid air is conducive to the hygroscopic growth of the aerosol particles, and some meteorological factors besides the wind field are detrimental to the diffusion and sedimentation of pollutants, thereby reducing the atmospheric Vis.

In this paper, we analyze the changes of atmospheric RH and wind field over ECN winters with $\mathrm{AO}$ in different phases to find out the relation between $\mathrm{AO}$ and the Vis.

The results of the study on Influence of AO on Winter Vis over ECN have some reference value for atmospheric Vis prediction and medium-long term forecast of haze. And it has important practical significance for social and economic development, the people's production and transportation safety of the region.

\section{Data and Methods}

The $\mathrm{RH}$ and wind field data (the resolution is $2.5^{\circ} \times 2.5^{\circ}$ ) were derived from the monthly-mean data of the National Center for Environmental Prediction and the Center for Atmospheric Research (NCEP/NCAR).

The AO Index monthly data is derived from the National Weather Service Climate Prediction Center.

The Vis data of ECN is extracted from the Global Summary of Day (GSOD).

\section{Influence of AO in Winter on the RH and Wind Field over ECN}

\subsection{Selection of the Month}

Using the AO index monthly data to draw December, January and February AO index line chart (Figure 1). 


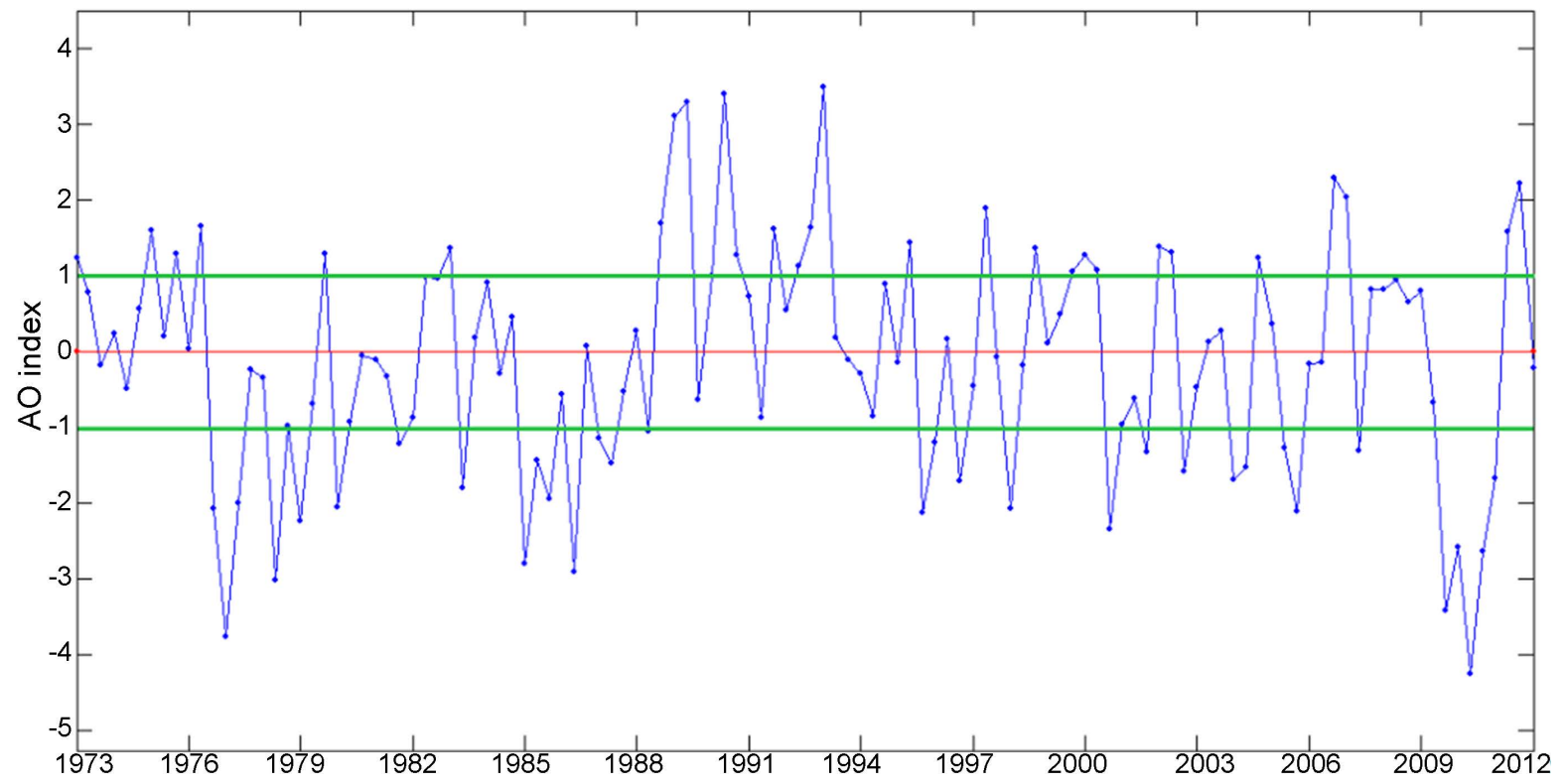

Figure 1. The trend of the arctic oscillation monthly index (December, January and February) from 1973 to 2012.

In this figure, the red line is zero and the two green lines are straight lines with $\mathrm{AO}$ index of 1 and -1 . Some typical months were selected as the positive AO month from those with $\mathrm{AO}$ index greater than 1 . And the negative $\mathrm{AO}$ month were selected from those with $\mathrm{AO}$ index less than -1 .

\subsection{Impact of AO in Winter on the RH and Wind Field}

The study of the RH and wind field with $\mathrm{AO}$ in different phases found that when the AO is in the positive phase (Figure 2(a)), the RH over ECN in winter is higher than the normal year. When the $\mathrm{AO}$ is in the negative phase (Figure 2(b)), a lower RH can be identified over ECN. In general, there is a negative correlation between Vis and RH. The lower RH leads to the improvement of Vis. This is because the more water vapor in the atmosphere is conducive to the hygroscopic growth of the aerosol particles, and the water vapor itself absorbs and scattered light [13]. Therefore, in the winters with a positive phase of AO, a higher $\mathrm{RH}$ can be identified over ECN, which is not conducive for Vis improvement. And the lower RH is favorable for Vis improvement when $\mathrm{AO}$ is in the negative phase.

From the wind field anomaly (Figure 2), when $\mathrm{AO}$ is in the positive phase, the wind anomaly is generally inshore in the coast areas of China, suggesting weakening of the East Asia winter monsoon associated with inefficient downwind transport, diffusion and dilution of the anthropogenic pollutants originated from ECN, which is consistent with Vis degradation over ECN. On the contrary, in the winters with a negative phase of $\mathrm{AO}$, the wind anomaly is generally offshore, the strengthened East Asia winter monsoon (associated with an enhancement of the downwind transport, diffusion and dilution of the pollutants) are favorable for Vis improvement. 


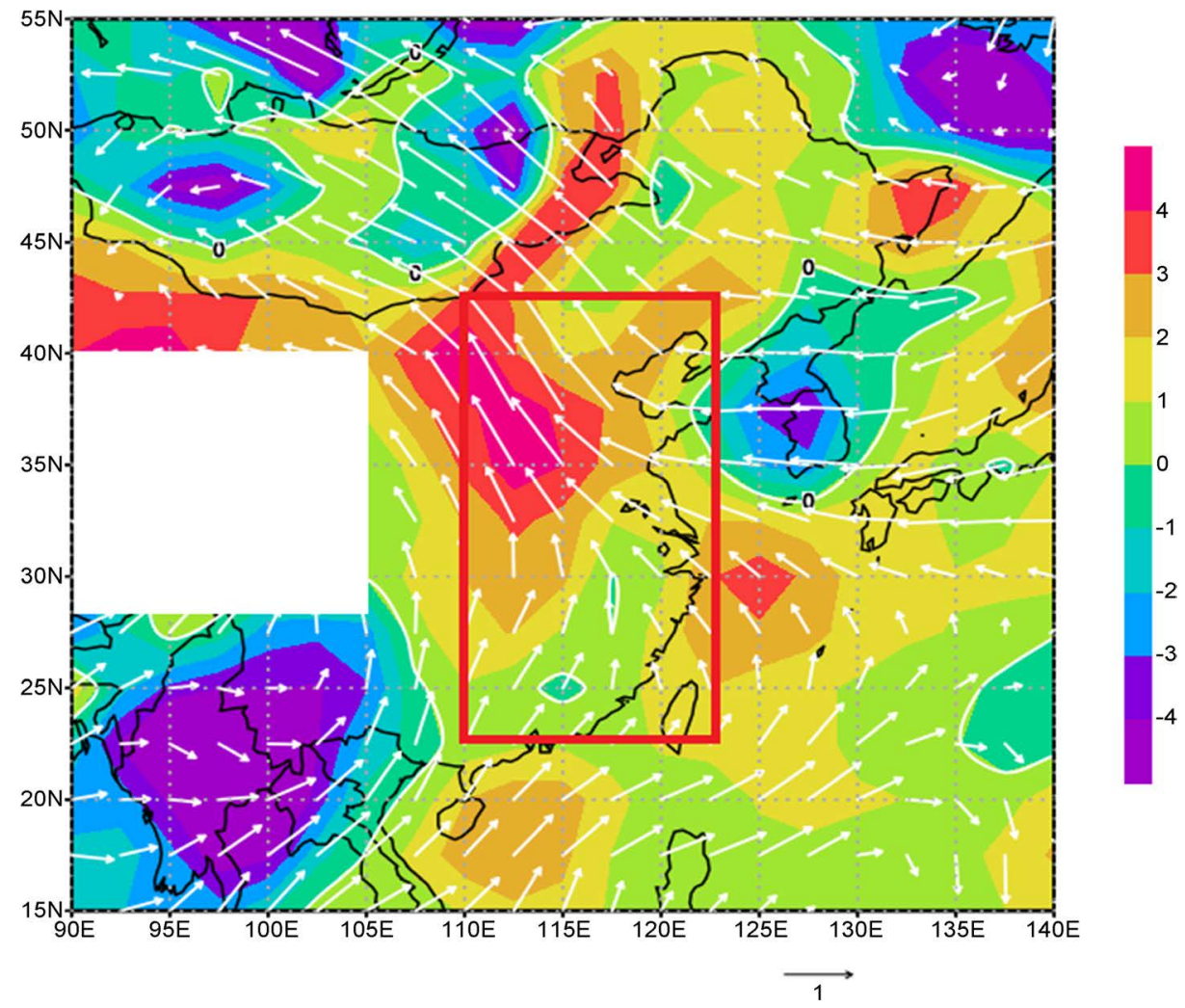

(a)

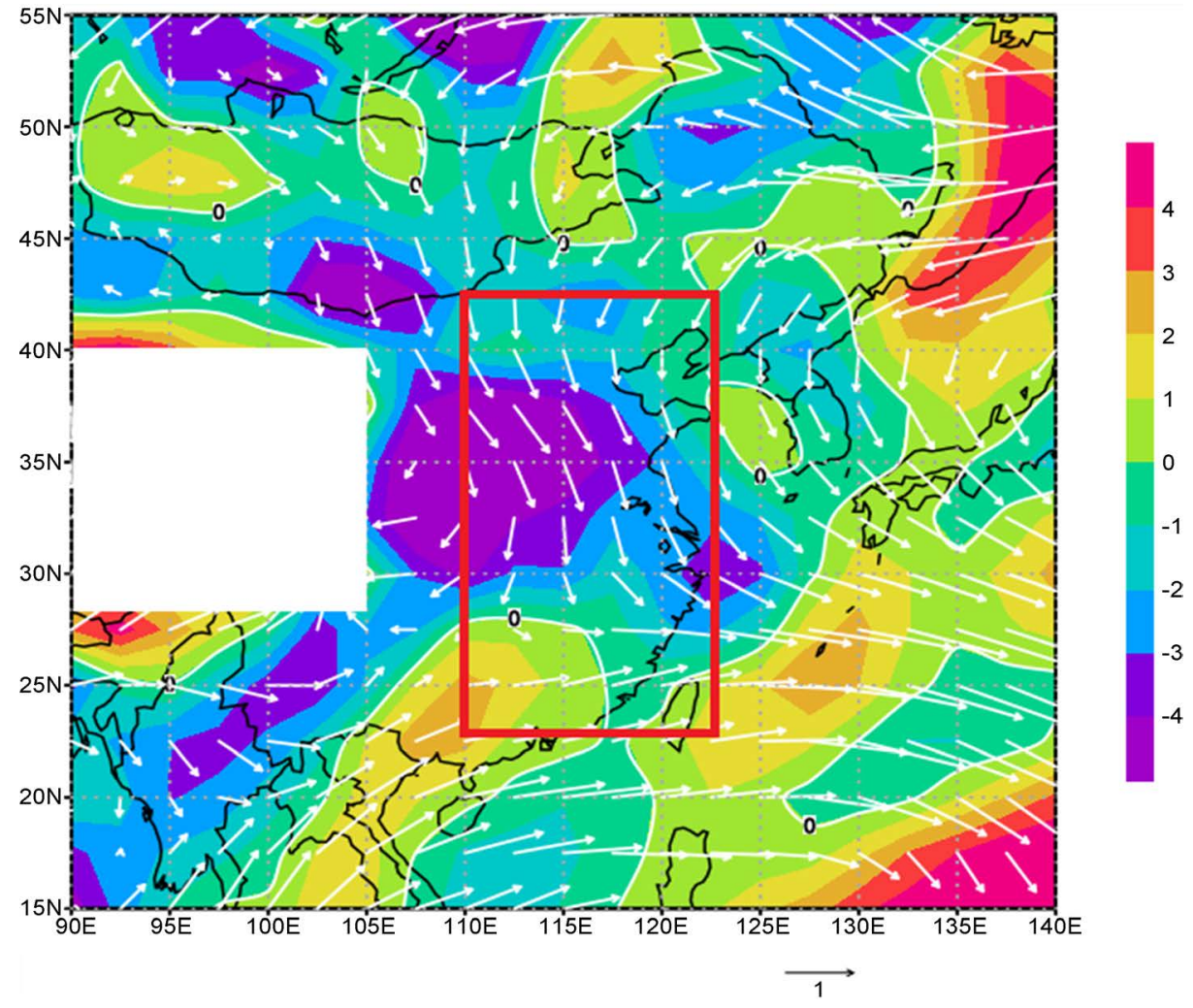

(b)

Figure 2. The wind fields and relative humidity anomaly over ECN in winter at $850 \mathrm{hPa}$ with the arctic oscillation in (a) positive phase and (b) negative phase. 


\section{Relationship between AO and Vis in ECN}

Because of the economic development and the increasing emission of anthropogenic pollutants, the Vis over ECN has declined year by year in recent 40 years [13]. The trend of average atmospheric Vis of 134 stations over ECN in the winter (December to the following year) from 1973 to 2012 was shown in Figure 3. The trend equation is

$$
y=-0.0665 x+10.864 .
$$

In order to study the influence of $\mathrm{AO}$ on the atmospheric Vis, we remove the factors of anthropogenic emissions. The Vis perturbation is obtained by subtracting the trend equation from the actual value of winter atmospheric Vis over ECN, then compared with the winter AO yearly index (after standardization), as shown in Figure 4. It can be seen that $\mathrm{AO}$ index is inversely proportional to winter Vis perturbation over $\mathrm{ECN}$, and the high values of $\mathrm{AO}$ index often correspond to the low values of Vis perturbation, while the low AO index correspond to the low Vis perturbation.

\section{Summary}

The influence of AO on Winter Vis over ECN is mainly reflected in: When $\mathrm{AO}$ is in the positive phase, the RH over ECN is higher than that in the normal year, which is favorable to the secondary formation and hygroscopic growth of the aerosol particles in the atmosphere. And the increase of the water vapor in the

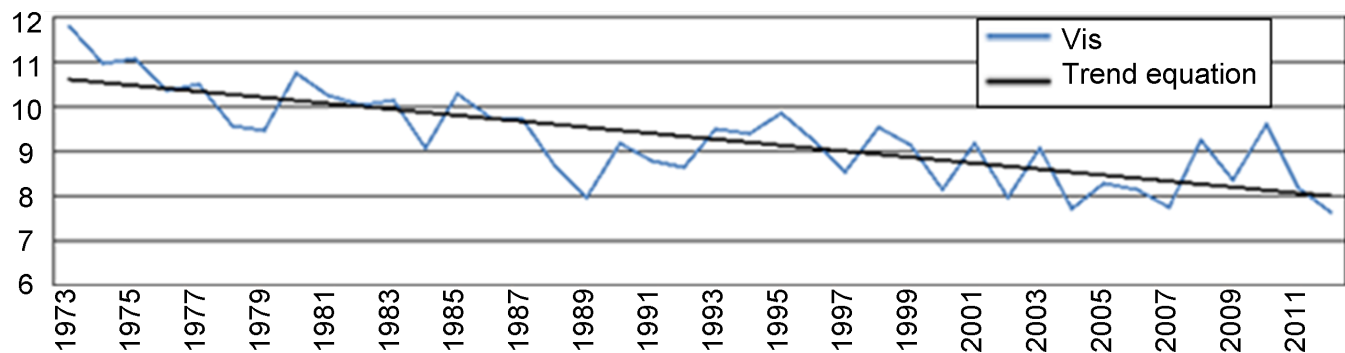

Figure 3. The trend of the visibility and the trend equation over ECN in winter from 1973 to 2012.

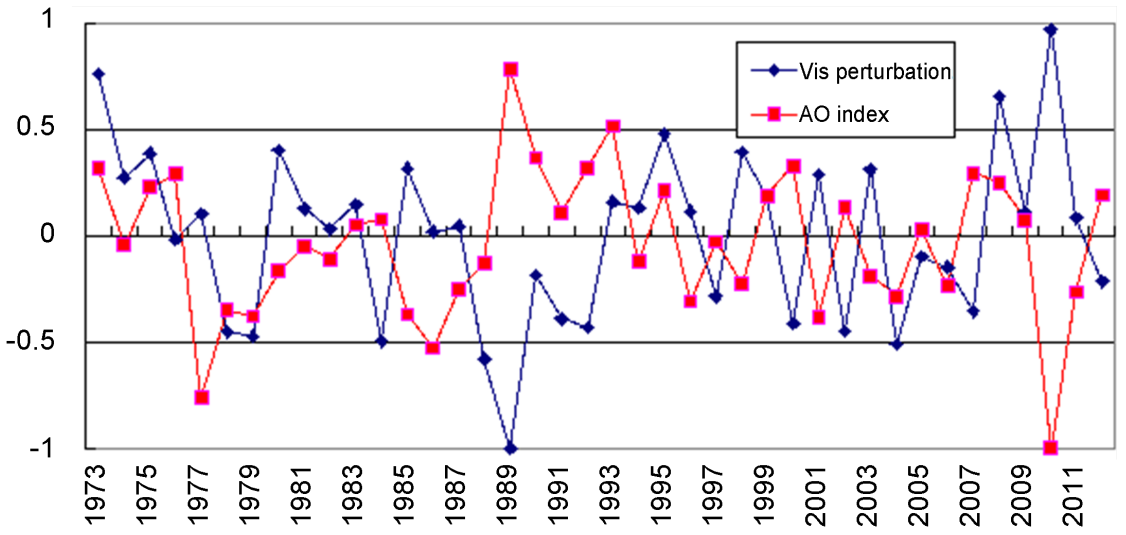

Figure 4. The trend of the visibility perturbation and the arctic oscillation index over ECN in winter from 1973 to 2012. 
atmosphere will enhance the absorption and scattering of light. The wind anomaly is generally inshore in the coast areas of China, suggesting weakening of the East Asia winter monsoon associated with inefficient downwind transport, diffusion and dilution of the anthropogenic pollutants originated from ECN, which is consistent with Vis degradation over ECN. On the contrary, in the winter with a negative phase of $\mathrm{AO}$, a lower RH can be identified over ECN, and the wind anomaly is generally offshore, which is favorable for Vis improvement.

Because of the economic development and the increasing emission of anthropogenic pollutants, the Vis over ECN has declined year by year in recent 40 years. AO index is inversely proportional to winter Vis perturbation over ECN, and the high values of $\mathrm{AO}$ index often correspond to the low values of Vis perturbation, while the low $\mathrm{AO}$ index corresponds to the low Vis perturbation.

The results of the study on influence of $\mathrm{AO}$ on winter Vis over ECN have some reference values for atmospheric Vis prediction and medium-long term forecast of haze.

\section{Acknowledgements}

Author thanks Dr. Qu Wenjun for the guidance and suggestions. This work was supported by Qingdao Bureau Research Subject for Youth (2016qdqxq9), Marine Public Welfare Project (20105018) and National Natural Science Foundation of China (41276012, 41430963).

\section{References}

[1] David, W.J., Thompson, John, M., et al. (1998) The Arctic Oscillation Signature in the Wintertime Geopotential Height and Temperature Fields. Geophysical Research Letters, 25, 1297-1300. https://doi.org/10.1029/98GL00950

[2] Ambaum, M.H.P., Hoskins, B.J. and Stephenson, D.B. (2001) Arctic Oscillation or North Atlantic Oscillation? Journal of Climate, 14, 3495-3507. https://doi.org/10.1175/1520-0442(2001)014<3495:AOONAO>2.0.CO;2

[3] Christiansen, B. (2002) On the Physical Nature of the Arctic Oscillation. Geophysical Research Letters, 29, 52-1-52-4. https://doi.org/10.1029/2002GL015208

[4] Baldwin, M.P. and Dunkerton, T.J. (1999) Propagation of the Arctic Oscillation from the Stratosphere to the Troposphere. Journal of Geophysical Research Atmospheres, 104, 30937-30946. https://doi.org/10.1029/1999JD900445

[5] Yang, X.Y., Jin, J.D., Zhou, Y., et al. (2007) Study on Arctic Oscillation Index and Its Interdecadal Variations. Guangzhou Meteorology, 3, 7-10.

[6] Qian, W.H. and Zhang, W.W. (2007) Cold Tide Time and Space Changes and Winter Warming in the Past 46 Years of China. Actomatics, 31, 1265-1278.

[7] Wei, F.Y. (2008) Changes of Cold Wave Disasters in China under the Background of Climate Change. Advances in Natural Science, 18, 289-295.

[8] Gong, D.Y. and Wang, S.W. (2003) Effects of Arctic Oscillation on Winter Climate of China in Recent 100 Years. Acta Geographica Sinica, 58, 559-568.

[9] He, C. and He, J.H. (2003) Study on the Relationship between Arctic Oscillation and Temperature in North China Winter. Journal of Nanjing Institute of Meteorology, 26, 1-7. 
[10] Zhang, H., Shi, C.E. and Qiu, M.Y. (2007) Effects of Meteorological Conditions on Atmospheric Visibility in Hefei. Journal of Atmospheric Sciences Research and Application, 2, 92-99.

[11] Wang, S.Y. and Xu, X.F. (2001) Influencing Factors of Atmospheric Visibility in Beijing. Academic Symposium on Meteorological Service of Urban Meteorology, 349-352.

[12] Shen, L.J., Wang, J.M., Li, P., et al. (2013) The Changes and Influencing Factors of Wuhan Atmospheric Visibility and Aerosol in January 2013. 2013 Chinese Environmental Science Conference, 5, 4661-4667.

[13] Zhang, L. and Wu, J. (2011) Analysis of the Changing Trend of Visibility in China from 1955 to 2000. Journal of Lanzhou University, 47, 46-55.

Submit or recommend next manuscript to SCIRP and we will provide best service for you:

Accepting pre-submission inquiries through Email, Facebook, LinkedIn, Twitter, etc. A wide selection of journals (inclusive of 9 subjects, more than 200 journals)

Providing 24-hour high-quality service

User-friendly online submission system

Fair and swift peer-review system

Efficient typesetting and proofreading procedure

Display of the result of downloads and visits, as well as the number of cited articles

Maximum dissemination of your research work

Submit your manuscript at: http://papersubmission.scirp.org/

Or contact gep@scirp.org 